\title{
Producción y tasa de descomposición de hojarasca en acahuales de Tabasco, México
}

\section{Production and litter decomposition rate in secondary vegetation of Tabasco, Mexico}

\author{
Antonio García-Domínguez', Luisa del Carmen Cámara Cabrales*, \\ Johannes Cornelis van der Wal² y José Luis Martínez Sánchez' \\ 1 Universidad Juárez Autónoma de Tabasco. \\ Villahermosa, Tabasco, México. \\ 2El Colegio de la Frontera Sur. Departamento de \\ Agricultura, Sociedad y Ambiente. Villahermosa, \\ Tabasco, México.
}

\section{RESUMEN}

La dinámica entre la caída y descomposición de hojarasca forma parte del funcionamiento de los ecosistemas. Se evaluó la producción, pérdida y tasa de descomposición de la hojarasca de selva y vegetación secundaria en unidades ecogeográficas (UE) de laderas de montañas, terrazas y planicies fluviales en la subregión de los Ríos de Tabasco en México. En cada UE se establecieron seis conglomerados con cuatro parcelas de $10 \mathrm{~m} \times 40 \mathrm{~m}$ y en tres parcelas de cada conglomerado se distribuyeron 6 trampas de $1 \mathrm{~m}^{2}(\mathrm{n}=144)$, las cuales se recogieron de marzo 2014 a marzo 2015 . Para determinar el peso remanente y la tasa de descomposición se utilizó el método de bolsas de descomposición, inicialmente con $10 \mathrm{~g}$ de hojarasca por bolsa ( $\mathrm{n}=384$ ). El periodo para determinar la descomposición estuvo comprendido de junio 2014 a marzo 2015 y mensualmente se recogieron cuatro bolsas por conglomerado, las cuales se secaron a peso constante. La producción de hojarasca en los acahuales de las UE fue similar a la de las zonas de vegetación primaria, excepto a la de una sola de estas últimas, que presentó mayor producción. La pérdida de peso y la tasa de descomposición fueron ligeramente mayores para vegetación primaria que para los acahuales. Los resultados encontrados sobre producción y degradación de la hojarasca pueden ser significativos por la inexistencia de trabajos en acahuales a escala de unidad ecogeográfica, lo que puede servir para darle mucha mayor importancia a este tipo de vegetación que asegure su conservación en el estado de Tabasco.

PALABRAS CLAVE: bolsas de descomposición, conglomerado, peso, secas, temporada, unidad ecogeográfica.

\section{ABSTRACT}

The dynamic between litter fall and its decay is part of the functioning of ecosystems. The production, loss and rate of decomposition of forest litter and secondary vegetation in ecogeographic units (EU) of mountain slopes, terraces and fluvial plains in the subregion of the rivers of Tabasco in Mexico were evaluated. In each EU, six conglomerates were established with four plots of $10 \mathrm{~m} \times 40 \mathrm{~m}$ and in three plots of each conglomerate, 6 traps of $1 \mathrm{~m}^{2}$ were distributed $(\mathrm{n}=144)$, which were collected from March 2014 to March 2015. To determine the remaining weight and the decomposition rate, the decomposition bags method was used, initially with $10 \mathrm{~g}$ of litter per bag $(n=384)$. The period to determine the decomposition was comprised from June 2014 to March 2015 and monthly, four bags were collected per conglomerate, which were dried at constant weight. The results show that the litter production of the secondary vegetation in the $\mathrm{EU}$ is similar to that of primary vegetation in two zones, while in only one of them it was higher. The weight loss and decomposition rate showed that, for primary vegetation, both are slightly higher than for the acabuales. These results may be significant due to the inexistent works about the production and decay of litter in the ecogeographic unit, that may help to secure the management and conservation of secondary vegetation in Tabasco state.

KEYWORDS: litterbags, conglomerate, weight, dried, season, ecogeographic unit. 


\section{INTRODUCCIÓN}

La caída y descomposición de hojarasca es un proceso de depósito y reducción de material orgánico que libera nutrientes al ecosistema para mantenerlo en funcionamiento (Vitousek et al., 1986; Polyakova y Billor, 2007). Esta dinámica que conlleva al reciclaje de nutrientes está precedida por procesos fisicoquímicos tales como trituración, lixiviación, catabolismo y humificación (RochaLoredo y Ramírez-Marcial, 2009; Singh y Gupta, 1977), gobernados generalmente por factores de suelo (Bonilla et al., 2008; Aceñolaza et al., 2010), clima (Coûteaux et al., 1995), calidad de hojarasca, abundancia de organismos degradadores (Lavelle et al., 1993; Aerts, 1997; Poggiani y Schumacher, 2004; Bautista y Delgado, 2006), etapa sucesional (Rivera-Vázquez et al., 2013), densidad arbórea y latitud (Reyes-Carrera et al., 2013).

La caída y descomposición de hojarasca se ha documentado que responde a patrones de temporalidad, variaciones ambientales de vientos, temperatura y precipitación (Álvarez-Sánchez, 2001), por lo que comúnmente en los zonas con alguna estacionalidad marcada, la caída de hojarasca puede hacerse más notoria en periodos más secos o bien en tiempos de máxima intensidad de lluvias (Ramírez y Ataroff, 2001; Ramírez et al., 2007; Quinto et al., 2007; Rivera-Vázquez et al., 2013), de igual forma se ha encontrado que una mayor tasa de descomposición se efectúa en periodos de alta humedad relacionada con la lluvia, lo que implica una mayor abundancia absoluta de organismos detritívoros (Bonilla et al., 2008; González y Seastedt, 2001), así mismo se ha demostrado que estas tasas de descomposición tienden a ser más altas en bosques tropicales lluviosos que en los secos, con una mayor descomposición en las zonas tropicales (Anderson y Swift, 1983; Del Valle, 2003).

Las caídas de hojarasca en los bosques primarios del mundo

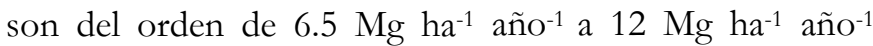
(Martius et al., 2004; Barlow et al., 2007), mientras que para la vegetación secundaria estas deposiciones mantienen un orden entre $8 \mathrm{Mg} \mathrm{ha}^{-1}$ año-1 y $11 \mathrm{Mg} \mathrm{ha}^{-1}$ año-1 (Hairiah et al., 2006; Schessl et al., 2008; Barlow et al., 2007). Su descomposición, en tanto, dependerá de las especies o mezcla de ellas, aunque la mayor pérdida de masa por descomposición se ha indicado que generalmente se llevara a cabo durante los tres primeros meses (Golley, 1978) Aunque a escala global (Monedero y González, 1995; Didham, 1998; McDonald y Healey, 2000; PalaciosBianchi, 2002; Di Stefano y Fournier, 2005; Caldato et al., 2010) y en México (Villavicencio-Enríquez, 2012; RiveraVázquez et al., 2013; Reyes-Carrera et al., 2013) existen estudios sobre tasas de producción y descomposición de hojarasca, puede afirmarse que son pocas las publicaciones realizadas en este país, para vegetación secundaria "acahuales" y selvas a lo largo de un gradiente altitudinal a escala de unidades ecogeográficas.

Este conocimiento de los procesos de producción, pérdida y descomposición de la hojarasca es información que puede dar soporte en un tiempo no muy lejano a políticas que conlleven a la conservación y manejo de los sistemas productivos forestales tropicales en el sureste tabasqueño, principalmente los derivados de vegetaciones primarias inmersas en el mosaico productivo de estos sitios.

\section{OBJETIVOS}

Determinar la producción, pérdida y tasa descomposición de hojarasca para dos tipos de vegetación; acahuales y selva mediana subperennifolia (SMSp) en tres unidades ecogeográficas con diferente altitud en la región de los Ríos, Tabasco, México.

\section{MATERIALES Y MÉTODOS}

\section{Área de estudio}

En la subregión de los Ríos, coordenadas 17²16’00” y $18^{\circ} 12^{\prime} 00^{\prime \prime}$ de latitud norte; $90^{\circ} 56^{\prime} 00^{\prime \prime}$ y $91^{\circ} 52^{\prime} 00^{\prime \prime}$ de longitud oeste, de los municipios de Tenosique, Balancán y Emiliano Zapata, del estado de Tabasco, México (Fig. 1), se identificaron tres unidades ecogeográficas (Ortiz-Pérez et al., 2005): las Laderas Septentrionales de la Sierra de Chiapas (LSSNCh) con altitud de $250 \mathrm{~m}$ snm, mantiene un clima cálido húmedo con lluvias todo el año (Af (m)), precipitaciones entre $2000 \mathrm{~mm}-4000 \mathrm{~mm}$ anuales y 

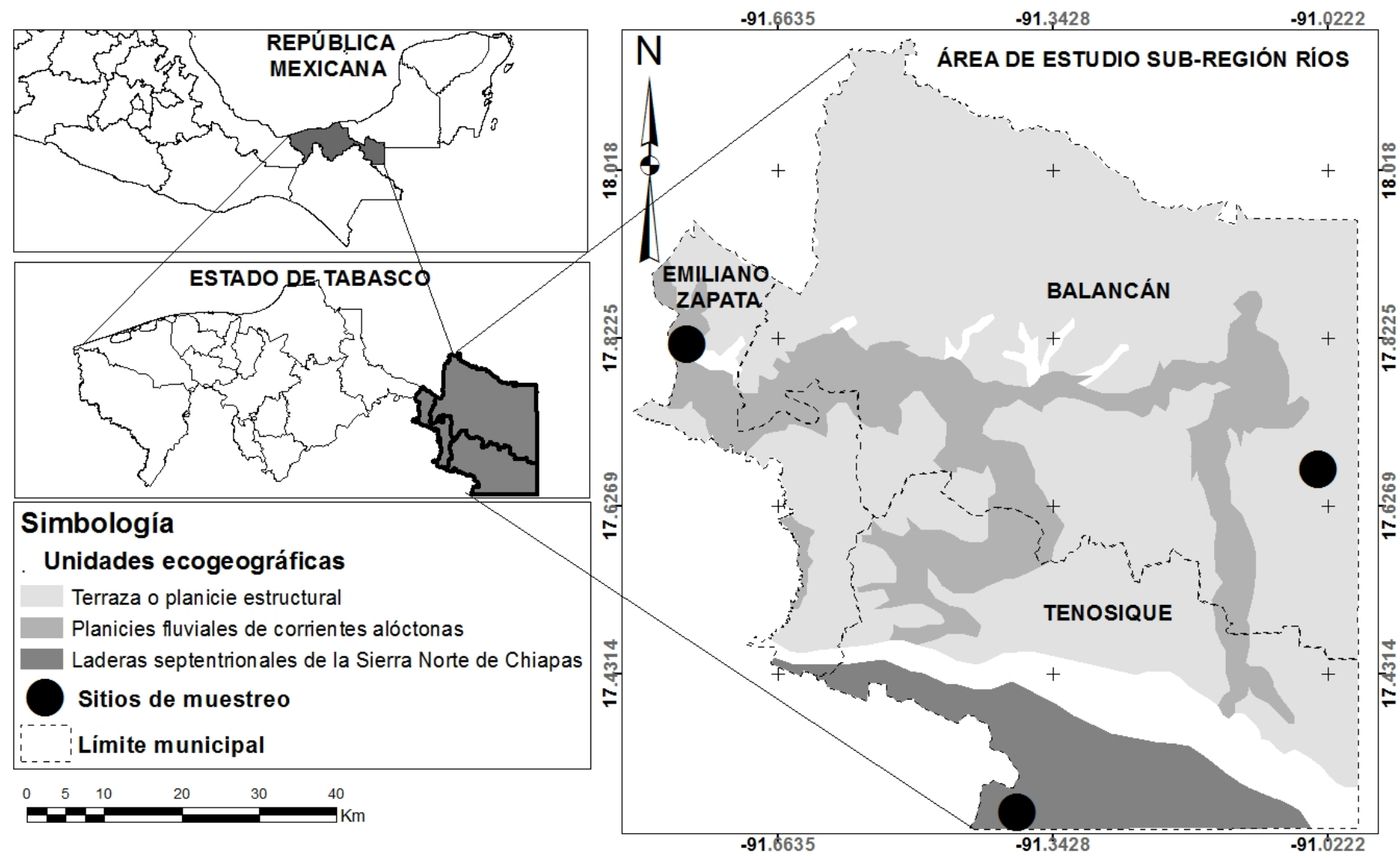

FIgURA 1. Unidades ecogeográficas (UE): Laderas Septentrionales de la Sierra Norte de Chiapas (LSSNCh) en Tenosique, Terrazas (T) en Balancán y Planicies Fluviales de Corrientes Alóctonas (PFCA) en Emiliano Zapata, en la región de los Ríos, Tabasco, México.

temperatura media anual que oscila de $22{ }^{\circ} \mathrm{C}$ a $29^{\circ} \mathrm{C}$, las Terrazas de Balancán (T) con $50 \mathrm{~m}$ snm, mantiene un clima cálido subhúmedo con lluvias en verano (Aw) precipitaciones que van de $1500 \mathrm{~mm}$ a $2000 \mathrm{~mm}$ y temperatura media anual de $26{ }^{\circ} \mathrm{C}$ y las Planicies Fluviales de Corrientes Alóctonas (PFCA) con $6 \mathrm{~m} \mathrm{snm}$, le corresponde un clima cálido húmedo con abundantes lluvias en verano (Am (f)), con $1500 \mathrm{~mm}$ a $2000 \mathrm{~mm}$ anuales de precipitación y temperatura media anual los $26{ }^{\circ} \mathrm{C}$ (García, 1973; Instituto Nacional de Estadística y Geografía [Inegi], 1986).

A partir de diferentes fuentes y pláticas personales con conocedores del tema y por la falta de bibliografía con referencia citable, se construyó la distribución de las temporadas o periodos de secas y lluvias para el estado de Tabasco. Se identificaron dos temporadas de sequías con poca precipitación: el primer periodo, conocido como seca, se encuentra identificado en los meses de marzo y abril; así mismo perteneciente a los meses secos existe una temporada conocida como canícula o sequía intraestival que abarca los últimos quince días del mes de julio y una gran parte del mes de agosto, la cual se caracteriza por días muy secos, altas temperaturas y una fase húmeda que provoca calores intensos.

Dentro de las temporadas de precipitación, se encuentra el periodo conocido como de lluvias que abarca los meses de mayo, junio, septiembre y octubre; en este periodo la precipitación se incrementa gradualmente, de medio lluvioso a torrenciales con corta duración. La última temporada, conocida como nortes, comprende los meses de noviembre, diciembre, enero y febrero; en ella se presentan 
nublados, disminución de la temperatura, lluvias de ligeras a fuertes con duración de varios días o semanas.

En las LSSNCh se seleccionaron dos localidades, los ejidos La Corregidora y Niños Héroes en el municipio de Tenosique, en los cuales se encontró la existencia de selva mediana subperennifolia (SMSp) con más de 100 años y acahuales derivados de las mismas con edades aproximadas de 30 años. De igual forma en $\mathrm{T}$ se seleccionaron dos localidades, ejido El Ramonal y El Capulín para el municipio de Balancán, con edades aproximadas de 15 años y 20 años de edad, respectivamente. Finalmente en PFCA tres localidades: El Avispero, El Pochote y El Jobal, en el municipio de Emiliano Zapata, los cuales comparten acahuales de una sola edad sucesional de aproximadamente 100 años, derivada de una histórica perturbación antropogénica (Tudela, 1989; Cámara-Cabrales et al., 2011; Rodríguez-Sandoval, 2012).

En cada ejido y por tipo de vegetación, se seleccionaron tres áreas completamente al azar en las cuales se establecieron tres conglomerados de $1600 \mathrm{~m}^{2}$, compuestos por cuatro parcelas de $10 \mathrm{~m} \times 40 \mathrm{~m}$ (Comisión Nacional Forestal [Conafor], 2010). En tres parcelas de cada conglomerado, se colocaron seis trampas para cuantificar la acumulación de hojarasca, resultando en $\mathrm{n}=$ 18 trampas por ejido (36 por UE para acahuales y 36 para SMSp) para un total de 144 trampas. Las trampas consistieron en bastidores de PVC de $1 \mathrm{~m}^{2}$ de área con tela mosquitera de $2 \mathrm{~mm}^{2}$ de luz, levantados a $50 \mathrm{~cm}$ del suelo. La hojarasca se recogió por un periodo de un año, en intervalos mensuales de marzo 2014 a marzo 2015. De cada trampa se obtuvo el peso húmedo in situ y el peso constante, después de someterse a secado en una estufa ce convección a $70{ }^{\circ} \mathrm{C}$. Para calcular la cantidad de hojarasca por unidad de área, el peso medio se extrapoló a una hectárea, expresándose los valores obtenidos en megagramos de hojarasca por hectárea por mes $\left(\mathrm{Mg} \mathrm{ha}^{-1} \mathrm{mes}^{-1}\right)$, así como megagramos por hectárea por año $\left(\mathrm{Mg} \mathrm{ha} \mathrm{a}^{-1}\right.$ año $\left.{ }^{-1}\right)$ considerándose como una producción total de hojarasca, sin distinción de componentes.

Para evaluar la pérdida y tasa de descomposición de hojarasca, se utilizó el método de bolsas de descomposición
(Litterbag method: Bärlocher, 2005 utilizado por RochaLoredo y Ramírez-Marcial, 2009). Las bolsas se elaboraron con malla de propileno (tela mosquitera) de $17 \mathrm{~cm} \times 25 \mathrm{~cm}$ de $2 \mathrm{~mm}^{2}$ de apertura, para un total de 48 bolsas por conglomerado (un total de 384 bolsas para todo el diseño experimental), estas contenían $10 \mathrm{~g}$ de mezcla homogénea de hojarasca seca a peso constante de la primera cosecha colectada de las seis trampas de cada conglomerado. Ocho bolsas de descomposición se colocaron superficialmente alrededor de las seis trampas recolectoras de hojarasca; mensualmente (de junio 2014 a marzo 2015) se retiraron cuatro bolsas por conglomerado, las cuales se llevaron al laboratorio para su secado en un horno de convección a 70 ${ }^{\circ} \mathrm{C}$ hasta lograr peso constante residual de cada muestra. Con lo anterior, se obtuvo el porcentaje de pérdida de necromasa inicial y final a cada tiempo.

El peso remanente se calculó mediante la ecuación:

$$
\% \mathrm{PR}=(X t / X o) * 100
$$

donde:

$\%$ PR: proporción de peso remanente

$X t$ : peso en el tiempo $t$ (días)

$X o:$ peso en el tiempo cero

La tasa de descomposición se calculó mediante la ecuación propuesta por Olson (1963):

$$
k=\frac{-\ln \left(\mathrm{X} / X_{0}\right)}{t}
$$

donde:

$k=$ tasa de descomposición

$X=$ peso de la hojarasca en un tiempo dado

$X_{0}=$ peso inicial de la hojarasca en el tiempo cero $t=$ tiempo (días)

Los datos de producción, pérdida y tasa de descomposición obtenidos se sometieron a un análisis de varianza de una vía o de un factor (Anova) para explorar diferencias entre las unidades ecogeográficas. Posteriormente, se les aplicó la prueba Tukey-Kramer HSD para encontrar diferencias estadísticas; ambas pruebas se realizaron $\operatorname{con} \alpha=0.05$. 
Estos análisis estuvieron apoyados con el paquete estadístico JMP® 8.0.2 y Excel 2016 ®.

\section{Resultados}

\section{Caída de hojarasca}

La deposición de hojarasca de la selva mediana subperennifolia (SMSp) en la unidad ecogeografica (UE) Laderas Septentrionales de la Sierra Norte de Chiapas

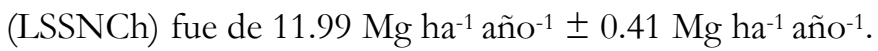

La mayor caída de hojarasca se observó en los meses de marzo, abril y julio, los dos primeros meses corresponden a la temporada de secas, con un promedio de $1.81 \mathrm{Mg} \mathrm{ha}^{-1}$ mes $^{-1} \pm 0.11 \mathrm{Mg} \mathrm{ha}^{-1} \mathrm{mes}^{-1}$ y $1.94 \mathrm{Mg} \mathrm{ha}{ }^{-1} \mathrm{mes}^{-1} \pm 0.13 \mathrm{Mg}$ $\mathrm{ha}^{-1} \mathrm{mes}^{-1}$ respectivamente, así como en julio dentro de la temporada conocida como canícula $\left(1.26 \mathrm{Mg} \mathrm{ha} \mathrm{mes}^{-1} \pm\right.$ $0.08 \mathrm{Mg} \mathrm{ha}^{-1} \mathrm{mes}^{-1}$ ), en ambas temporadas la precipitación es poca, de acuerdo con los históricos en estos territorios. Los acahuales en LSSNCh, presentaron una caida de hojarasca de $8.88 \mathrm{Mg} \mathrm{ha}^{-1}$ año-1 $\pm 0.51 \mathrm{Mg} \mathrm{ha}^{-1}$ año $^{-1}$. La distribución mensual en el año de la caída de hojarasca en LSSNCh para los acahuales mantuvo un patrón semejante a lo observado en SMSp. Sin embargo, el mes de deposición máxima de los acahuales con respecto al mes de deposición máxima de la SMSp en LSSNCh fue menor a $0.5 \mathrm{Mg} \mathrm{ha}^{-1}$ mes $^{-1}$ (Fig.2).

En $T$, la producción de hojarasca para los acahuales fue de $14.20 \mathrm{Mg} \mathrm{ha}^{-1}$ año ${ }^{-1} \pm 0.40 \mathrm{Mg} \mathrm{ha}^{-1}$ año $^{-1}$. La mayor deposición de hojarasca se presentó en los meses de abril en 2014 y marzo 2015, aunque en enero y febrero también puede observarse estos picos de caída máxima. En T, el patrón de caída fue distinto al encontrado en LSSNCh, tanto en SMSp como en acahuales, ya que pueden observarse caídas máximas en enero y febrero meses correspondientes a la temporada de nortes, donde existen altos índices de precipitación (Fig. 2).

En los acahuales de las Planicies Fluviales de Corrientes Aloctonas (PFCA) la caída de hojarasca registrada fue de $7.68 \mathrm{Mg} \mathrm{ha}^{-1} \mathrm{año}^{-1} \pm 0.57 \mathrm{Mg} \mathrm{ha}^{-1} \mathrm{año}^{-1}$. La máxima caída se presentó en marzo 2015; temporada de seca y en julio temporada conocida como canícula, ambas temporadas de escasa precicitación. En T, se presentó en el mes de noviembre que no pertenece a los meses secos (Fig. 2).

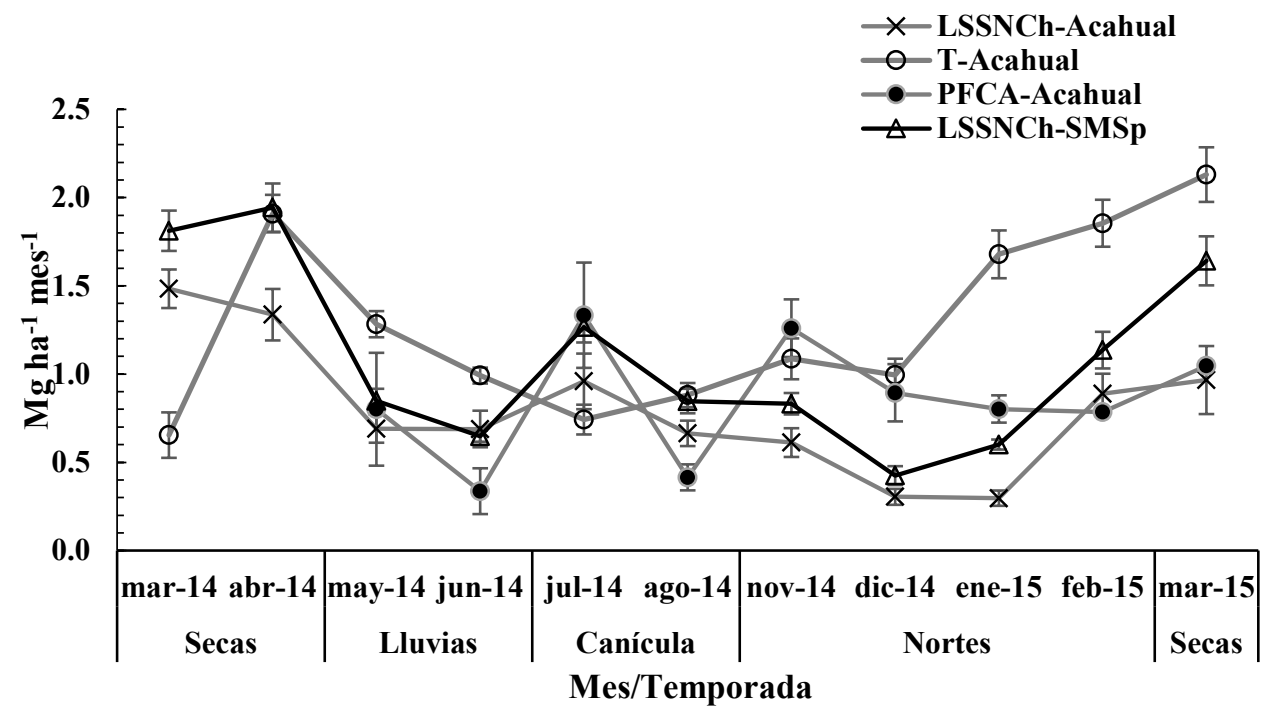

FIgurA 2. Caída mensual de hojarasca (promedio \pm e. e., $\mathrm{Mg}_{\mathrm{ha}} \mathrm{ha}^{-1} \mathrm{mes}^{-1}$ ) durante el periodo marzo de 2014 marzo de 2015, en las UE; Laderas Septentrionales de la Sierra Norte de Chiapas (LSSNCh) en vegetación de Selva Mediana Subperennifolia (SMSp) y de los acahuales en las Laderas Septentrionales de la Sierra Norte de Chiapas (LSSNCh), Terrazas de Balancán (T) y Planicies Fluviales de Corrientes Alóctonas (PFCA). 
El Anova realizado en las LSSNCh-SMSp, LSSNChAcahual, T-Acahual y PFCA-Acahual mostraron que en la deposicon mensual y entre temporadas en cada unidad ecogeogeografica existió diferencias altamente significativa $(\alpha<0.05)$ para la producción de hojarasca.

La prueba de Tukey-Kramer HSD reveló que entre temporadas para LSSNCh-SMSp y acahuales, la seca mantiene diferencia estadistica sobre las otras temporadas, mientras que lluvias y nortes son similares en cuanto a esta caida de hojarasca. La misma prueba mostró que, en T, solo las temporadas de secas y canícula fueron diferentes. En las PFCA, la temporada de nortes y secas mantuvieron difrencia significativa $(\alpha<0.05)$.

Los coeficientes de correlación de Pearson entre producción mensual de hojarasca y precipitación promedio mensual efectuados en LSSNCh para SMSp $(r=0.250)$ y Acahual $(\mathrm{r}=0.191), \mathrm{T}$ acahual $(\mathrm{r}=0.018)$ y PFCA acahual $(\mathrm{r}=0.396)$, a una $\mathrm{P}<0.05$, mostraron que no existe una correlación fuerte con la precipitación.

\section{Peso remanente}

Después de ocho meses de haber puesto la hojarasca en cada una de las regiones ecogeográficas para su descomposición, de manera general se observó una disminución continua del peso inicial a lo largo del tiempo. En los primeros 30 días, en SMSp y acahuales de la LSSNCh, se registró una pérdida del peso inicial de 20\%, mientras que, para los acahuales en T, esta disminución de peso fue del 15\%. La prueba de Tukey-Kramer HSD, $(\alpha<$ $0.05)$ indicó que la pérdida de peso de cada UE, para este primer mes no difieren significativamente. A partir del segundo y hasta el cuarto mes, la prueba a posteriori mostró que sí existió diferencia significativa entre tipos de vegetación, y que es la SMSp la que registró en general un mayor porcentaje de perdida de hojarasca en cada uno de estos meses. Los acahuales de ambas unidades ecogeográficas, no presentan diferencia en el porcentaje de pérdida, al compararse de forma mensual. En la SMSp, la pérdida del 50\% de su peso inicial se registró en 180 días después de su puesta en la UE, mientras que en los acahuales para LSSNCh y T, este mismo porcentaje se presentó a los 265 días (Fig. 3).

\section{Tasa de descomposición}

En todas las unidades ecogeográficas, la velocidad de descomposición se presentó de manera generalizada en una forma decreciente con el trascurso del tiempo para los dos tipos de vegetación en estudio. Los valores obtenidos para la tasa de descomposición, durante el primer mes, son relativamente más altos que en los siguientes meses. $\mathrm{Al}$ ser comparadas las velocidades de descomposición entre unidades ecogeográficas, para este primer intervalo de tiempo, la prueba de Tukey-Kramer HSD $(\alpha<0.05)$ advirtió que estas, son similares para los primeros 30 días.

Las velocidades de descomposición en cada una de las UE se mantienen de forma decreciente con un mayor dinamismo en los tres primeros meses, ya que partir del cuarto mes, esta velocidad disminuye y se manifiesta de forma lineal con el paso del tiempo. Al ser comparadas las velocidades de descomposición entre tipos de vegetación (Tukey-Kramer HSD, $\alpha<0.05$ ), la SMSp a partir del segundo mes, es la que sostiene velocidades superiores de descomposición y que los acahuales sin importar la unidad ecogeográfica, no son diferentes para los mismos meses.

\section{DISCUSIÓN}

La producción de hojarasca en la SMSp en LSSNCh, estuvo dentro del intervalo señalado por Martius et al. (2004) y Barlow et al. (2007), que es de $6.5 \mathrm{Mg} \mathrm{ha}^{-1}$ año-1 a $12 \mathrm{Mg}$ ha1 año ${ }^{-1}$, quienes podrían considerarse como referente en cuanto a la producción de hojarasca a nivel mundial, así mismo fue ligeramente superior a la registrada por Didman (1998) de $7.948 \mathrm{Mg} \mathrm{ha}^{-1}$ año-1 $^{-1}$ McDonald y Healey (2000) de $9.319 \mathrm{Mg} \mathrm{ha}^{-1}$ año $^{-1}$, Palacios-Bianchi (2002) de $8.36 \mathrm{Mg}$ ha $^{-1}$ año $^{-1}$, Villavicencio-Enríquez (2012) de $8.190 \mathrm{Mg} \mathrm{ha}^{-1}$ año-1, pero similar a lo encontrado por Monedero y González (1995): $10.7 \mathrm{Mg} \mathrm{ha}^{-1}$ año-1 $^{-1}$ Los acahuales en LSSNCh y de las PFCA estuvieron dentro del intervalo de $8 \mathrm{Mg} \mathrm{ha}^{-1}$ año $^{-1}$ a $11 \mathrm{Mg} \mathrm{ha}^{-1}$ año $^{-1}$ presentados por Hairiah et al. (2006); Schessl et al. (2008) y Barlow et al. (2007), para vegetación secundaria como un referente de producción de 
hojarasca. La producción de hojarasca en T para acahuales fue superior a este intervalo, inclusive para vegetación primaria. Esta deposición en T representa 46\% del total de la hojarasca para acahuales de la subregión en estudio.

La existencia de una mayor deposición de hojarasca en la unidad ecogeográfica $\mathrm{T}$, mantiene relación con lo encontrado por Rivera-Vázquez et al. (2013), Rave-Oviedo (2013) y Wang et al. (2007). Estos autores hallaron que estados sucesionales jóvenes y en pleno crecimiento menores a 20 años, este tipo de vegetación mantienen una mayor caída de hojarasca que sucesiones de mayor edad, característica de menor edad estuvo presente en la unidad ecogeográfica terrazas.

Otra cualidad relacionada con una alta productividad de hojarasca también fue descrita por Neiff y de Neiff (1990) y Aceñolaza et al. (2010), estos argumentan que una mayor densidad en rodales jóvenes también hace posible una mayor producción de hojarasca. En T, a diferencia de las otras unidades existió una mayor densidad de individuos (877 Ind. ha-1), en LSSNCh para acahuales fue de 810 Ind. $\mathrm{ha}^{-1}$ mientras que para selvas fue de $776 \mathrm{Ind} \mathrm{ha}^{-1} \mathrm{y}$ para PFCA de 406 Ind. ha-1.

Aunque no existen estudios sobre si la proporción de individuos de árboles jóvenes con respecto a árboles adultos en acahuales para rodales mixtos incida en la producción de hojarasca (considerando los jóvenes de diámetros $\geq 2.5 \mathrm{y} \leq 10 \mathrm{~cm}$ de diámetro normal; DN y adultos a individuos $\geq 10 \mathrm{~cm}$ de $\mathrm{DN}$ ); se observa una proporción del $50 \%$ de jóvenes sobre adultos en la unidad ecogeográfica $\mathrm{T}$ a diferencia del $30 \%$ en otras unidades (García-Domínguez et al. 2018), se puede pensar que esta condición esté coadyuvando a una producción mayor de hojarasca por encima de las demás. Este comportamiento con referencia al DN diferenciando entre rodales con individuos jóvenes o adultos, así como su densidad, se ha observado en plantaciones estudiadas por Jeong et al. (2009), donde rodales con mayor densidad y menores DN han mantenido una producción mayor de hojarasca, por encima de menor densidad y mayores diámetros.

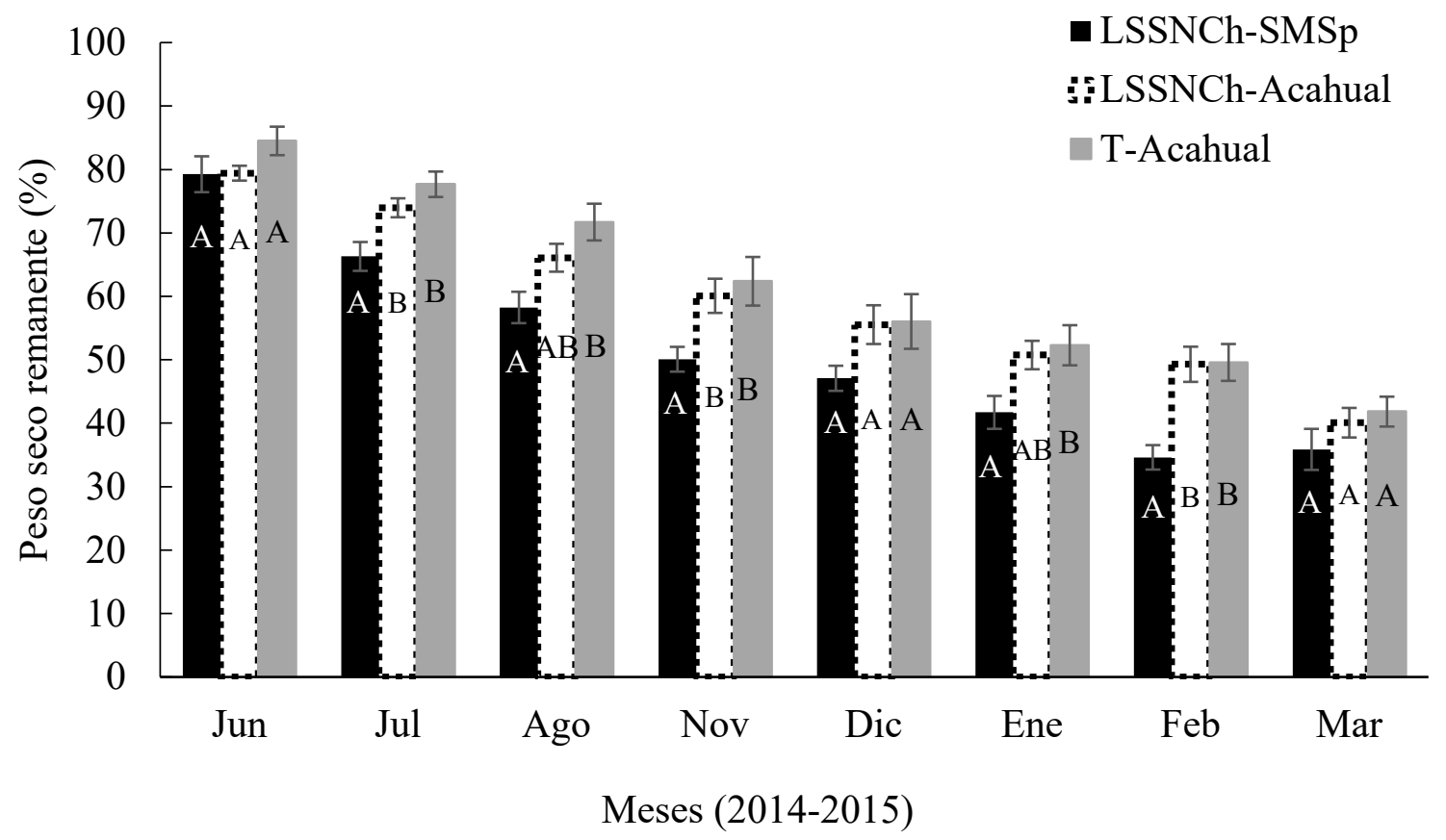

FIGURA 3. Porcentaje de peso seco remanente de hojarasca (promedio \pm e.e, intervalo de confianza del 95\%) en bolsas de descomposición en dos unidades ecogeográficas LSSNCh y T para dos tipos de vegetación selvas y acahuales.

Las letras dentro de las barras indican diferencias entre cada mes $(P \leq 0.05)$ con la prueba a posteriori de Tukey-Kramer HSD. 


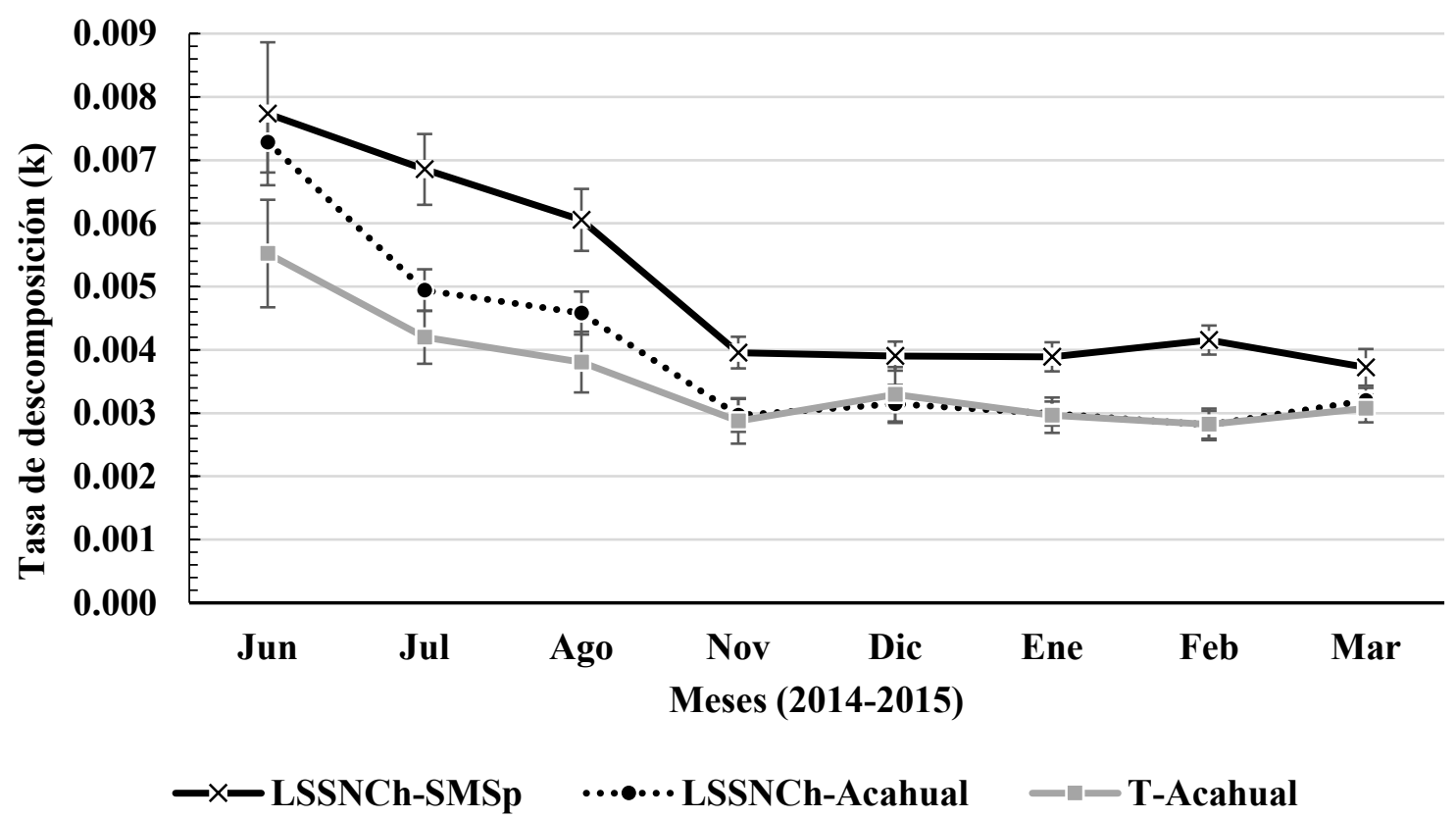

FIGURA 4. Tasa de descomposición (k) de la hojarasca (promedio \pm e. e.) en selva mediana subperennifolia (SMSp) y acahuales, para las unidades ecogeográficas Laderas Septentrionales de la Sierra Norte de Chiapas (LSSNCh), Terrazas de Balancán (T).

El comportamiento de caída de hojarasca en la unidad ecogeográfica LSSNCh para los dos tipos de vegetación se ajusta a lo que típicamente se ha registrado (Golley, 1983; Álvarez-Sánchez y Guevara, 1993; Di Stefano y Fournier, 2005; Orihuela-Belmonte et al., 2012; Rivera-Vázquez et al. (2013); Rave-Oviedo, 2013), que es una mayor caída de hojarasca en periodos de sequías (secas y canícula) como estrategia de la vegetación para evitar perdida de agua, lo que favorece una abundante caída de material foliar. Inversamente a este comportamiento típico, en las unidades ecogeográficas T y PFCA, se observaron picos de máxima caída en periodos de lluvias y nortes, comportamiento reportado por Sarmiento (1984); Veneklaas (1991); Rodríguez y Rosas (1993); Caldato et al. (2010) (Fig. 2).

Un factor más por el cual se esté dando un máximo de producción de hojarasca en $\mathrm{T}$ puede estar relacionado con el hecho que dicha unidad ecogeográfica se encuentra enclavada en la porción más seca del territorio tabasqueño (Cámara-Cabrales et al., 2011).

La pérdida de peso a través del tiempo y la tasa de descomposición en cada una de las regiones ecogeográficas mantiene un comportamiento similar al descrito por Arellano et al. (2004) y Villavicencio-Enríquez (2012), quienes manifiestan que la hojarasca debe de estar sometida a dos fases de degradación para darle cabida al proceso de reducción; la primera conocida como fase inicial que es de rápido desarrollo. Ahí se propicia la pérdida de materiales de fácil degradación en lapsos de tiempo cortos, lo que provoca que un mayor porcentaje de peso se pierda y la velocidad de descomposición aumente en un tiempo breve, como pudo observarse en los valores obtenidos en este estudio (Fig. 3 y 4).

Así mismo, con los datos encontrados, también puede inferirse que existió una segunda fase de descomposición, donde el porcentaje de pérdida de hojarasca disminuyó y de igual forma su tasa. También pudo observarse que este comportamiento requirió un tiempo mayor para mostrar descomposición y pérdida de peso como en la fase inicial. Esta fase lenta, según lo señalado por la bibliografía, se debe a que la mayoría de los elementos restantes a degradar son del tipo recalcitrantes, que al volverse más estables repercuten en un menor porcentaje de pérdida de peso y 
una tasa de descomposición de igual forma menor. Lo que requerirá mayores lapsos de tiempo para lograr la degradación de estos elementos (Gómez y Sánchez, 1999; Arellano et al., 2004; Goma-Tchimbakala y BernhardReversat, 2006). Este comportamiento también fue congruente con los resultados presentados por Swift y Anderson (1989); Xuluc-Tolosa et al., (2003); Fioretto et al., (2005).

El menor o mayor tiempo requerido para que la pérdida de peso alcanzara $50 \%$ en la selva y en los acahuales probablemente se deba a factores propios de cada ecosistema (Swift et al., 1979; Aerts y Chapin, 2000); es sabido que en el caso de las selvas (Álvarez-Sánchez y Naranjo-García, 2003) al existir una mayor humedad y altas temperaturas, la macrofauna, la actividad microbiana y la aparición de microflora, tales como hongos, pueden favorecer altamente a la descomposición de estructuras moleculares más solubles que se encuentran presentes en la necromasa foliar, por lo que menores tiempos para la degradación pueden estar favoreciendo la pérdida de peso y aumentando la velocidad de descomposición. Así mismo, en sitios perturbados como los acahuales donde la pérdida de humedad podría considerarse constante por la poca cobertura arbórea, el número de poblaciones microbianas y de hongos pueden ser pequeño en comparación con las selvas, lo que probablemente este repercutiendo en menores porcentajes de pérdida de peso e inferiores velocidades de descomposición, lo cual va a requerir tiempos mucho más largos para lograr la degradación para estos ecosistemas. En el caso de selvas, pérdidas mayores de peso y altas tasas de descomposición también se encuentran relacionadas íntimamente con la calidad de la hojarasca, registros como los de Álvarez-Sánchez y Becerra (1996) han indicado que estos ecosistemas han logrado descomponer la hojarasca hasta 30\% más rápido por encima de otros ecosistemas, como pudo observarse al contrastar selvas y acahuales (Fig. 3 y 4).

\section{CONCLUSIONES}

La caída de hojarasca de selvas y acahuales, en general, mantiene una producción similar a lo registrado para bosques tropicales húmedos del mundo. Aunque típicamente se habría esperado una mayor caída de hojarasca, en relación con la poca precipitación (mayor en temporadas secas). En este estudio se encontró que caídas máximas también coexisten en periodos con meses donde no necesariamente existe un déficit de lluvias a la cual se refiere como temporada de nortes.

En lo que a pérdida de peso se refiere a través del tiempo, así como las tasas de descomposición en todas las unidades ecogeográficas, mantuvieron un comportamiento similar. En la vegetación primaria de SMSp existió menor peso remanente y mayores tasas de descomposición, como se ha demostrado en otros estudios para bosques tropicales, a diferencia de los acahuales donde el peso remanente es mayor y sus tasas de descomposición fueron menores. Hay que mencionar que ningún trabajo en el sureste mexicano se ha hecho con tres regiones que intenten mostrar que los acahuales en estos sitios pueden representar un potencial para el manejo y conservación de estos.

Aunque en este estudio no se haya evaluado el factor edad como variable que influye en producción de hojarasca, se pudo observar que una mayor producción se dio en la unidad ecogeográfica $T$, que es donde convergen acahuales de menor edad o etapas sucesionales jóvenes.

\section{RECONOCIMIENTOS}

Al Consejo Nacional de Ciencia y Tecnología (Conacyt) por la beca otorgada para estudios de posgrado. Este artículo es parte de los productos del proyecto de investigación doctoral "Determinación de la biomasa en vegetación de los sistemas de producción forestal en la cuenca baja del Usumacinta en la región de los Ríos, Tabasco, México" del proyecto FOMIX de cambio global y sustentabilidad en la cuenca del Usumacinta, del Centro del Cambio Global y la 
Sustentabilidad en el Sureste A.C. (CCGSS) TAB-2012-C28-194316 "Retos para la sustentabilidad en la cuenca del Río Usumacinta en Tabasco: Ecosistemas, Cambio Climático y Respuesta Social.”, Ecosur y UJAT. Así mismo a todos los que participaron en las diversas etapas de este estudio, especialmente a los productores de los ejidos Niños Héroes, Corregidora, Capulín, Ramonal, Jobal, Avispero y Pochote.

\section{REFERENCIAS}

Aceñolaza, P. G., Zamboni, L. P., Rodríguez, E. E. \& Gallardo, J. F. (2010). Litterfall production in forests located at the Pre-delta area of the Paraná River (Argentina). Annals of Forest Science, 67(311), 110. doi: $10.1051 /$ forest $/ 2009117$

Aerts, R. (1997). Climate, leaf litter chemistry and leaf litter decomposition in terrestrial ecosystems: a triangular relationship. Oikos, 79, 439-449. doi: 10.2307/3546886

Aerts, R. \& Chapin, F. S. (2000). The mineral nutrition of wild plants revisited: a re-evaluation of processes and patterns. Advances in Ecological Research. 30, 1-67. doi:10.1016/S0065-2504(08)60016-1

Álvarez-Sánchez, J. (2001). Descomposición y ciclo de nutrientes en Ecosistemas terrestres de México. Acta Zoológica Mexicana (nueva serie). Número especial, 1, 11-27. Recuperado de http://www.redalyc.org/pdf/575/57500002.pdf

Álvarez-Sánchez, J. \& Becerra, R. (1996). Leaf decomposition in a Mexican tropical rain forest. Biotropica, 28(4b), 657-667. doi: $10.2307 / 2389052$

Álvarez-Sánchez, J. \& Guevara, S. (1993). Litterfall dynamics in a Mexican lowland tropical rain forest. Tropical Ecology, 34(2), 127142.

Álvarez-Sánchez, J. \& Naranjo-García, E. (Eds.). (2003). Ecología del suelo en la selva tropical húmeda de México. Instituto de Ecología, A. C., Instituto de Biología y Facultad de Ciencias, UNAM. Xalapa, México.

Anderson J. \& Swift, M. (1983). Decomposition in tropical forests. En S. L. Sutton., T. C. Whitmore \& A. C. Chadwick (Eds.), Tropical rain forest: ecology and management (pp. 287-327). Oxford, U. K.: Blackwell.

Arellano, R., Paolini, J., Vásquez, L. \& Mora, E. (2004). Producción y descomposición de hojarasca en tres agroecosistemas de café en el estado de Trujillo, Venezuela. Revista Forestal Venezolana, 48, 714.
Bärlocher, F. (2005). Leaf mass loss estimated by litter bag technique. En A. S. Graca., F, Bärlocher y M. O. Gessner (Eds.), Methods to study litter decomposition: a practical guide (pp. 36-42). Dordrecht, Alemania: Springer. Recuperado de https://www.researchgate.net/profile/Felix_Baerlocher/publica tion/226372640_Leaf_Mass_Loss_Estimated_by_Litter_Bag_T echnique/links/0fcfd50abd2e088fad000000.pdf

Barlow, J., Gardner, T. A., Ferreira, L.V. \& Peres, C. A. (2007). Litter fall and decomposition in primary, secondary and plantation forests in the Brazilian Amazon. Forest Ecology and Management, 247(1-3), 91-97. doi: 10.1016/j.foreco.2007.04.017

Bautista, F. \& Delgado, C. (2005). Descomposición de hojarasca y abundancia de macroinvertebrados por el uso de mantillos y cultivos de cobertera. En F. Bautista y G. Palacio (Eds.), Caracterización y manejo de los suelos de la península de Yucatán: Implicaciones Agropecuarias, Forestales y Ambientales (pp. 269-281). Universidad Autónoma de Campeche, Universidad Autónoma de Yucatán. Campeche, Campeche, México.

Bonilla, R., Belisario-Roncallo, J. J. \& García, T. (2008). Producción y descomposición de la hojarasca en bosques nativos y de Leucaena sp., en Codazzi, Cesar. Revista Corpoica - Ciencia y Tecnología Agropecuaria, 9(2), 5-11.

Caldato, S. L., Floss, P. A. \& Serafini, F. E. C. (2010). Producción y descomposición de hojarasca en la selva ombrófila mixta en el sur de Brasil. Bosque (V aldivia), 31(1), 3-8.

Cámara-Cabrales, L. C., Hernández-Trejo, H., Castillo-Acosta, O., Galindo-Alcántara, A., Morales, A., Zequeira-Larios, C., ... \& Guadarrama, M. A. (2011). Estudio regional de la UMAFOR de los Ríos. Informe técnico. UJAT-DACBiol. Conafor-Unión de silvicultores. Villahermosa, Tabasco, México.

Comisión Nacional Forestal [Conafor] (2010). Inventario nacional forestal y de suelos: Manual y procedimientos para el muestreo de campo Re-muestreo. Recuperado de http://www.climateactionreserve.org/wp content/uploads/2011/03/ Sampling_Manual-_Remuestreo_Conafor_INFyS.pdf

Coûteaux, M. M., Bottner, P. \& Berg, B. (1995). Litter decomposition, climate and liter quality. Trends in Ecology and Evolution, 10(2), 63 66. doi: 10.1016/S0169-5347(00)88978-8

Del Valle-Arango, J. I. (2003). Descomposición de la hojarasca fina en bosques pantanosos del Pacífico Colombiano. Interciencia, 28(3), 148-153.

Di Stefano, J. F., \& Fournier, L. A. (2005). Caída de hojarasca y tasas de descomposición de las hojas de Vochysia guatemalesis en una 
plantación de 10 años, Tabarcia de Mora, Costa Rica. Agronomía Costarricense, 29(1), 9-16.

Didham, R. K. (1998). Altered leaf-litter decomposition rates in tropical forest fragments. Oecologia, 116(3), 397-406. doi: $10.1007 / \mathrm{s} 004420050603$

Fioretto, A., Dinardo, C., Papa, S \& Fuggi, A. (2005). Lignin and cellulose degradation and nitrogen dynamics during decomposition of three leaf litter species in a Mediterranean ecosystem. Soil Biology \& Biochemistry, 37(6), 1083-1091. doi: $10.1016 /$ j.soilbio.2004.11.007

García, E. (1973). Modificaciones al sistema de clasificación climática de Köppen (2a ed.). México: Universidad Nacional Autónoma de México, Instituto de Geografía.

García-Domínguez, A., Cámara, C. L. C., Van der, W. J. C. \& Martínez, Z. P. (2018). Biomasa en acahuales de tres unidades ecogeográficas del estado de Tabasco. Revista Mexicana de Ciencias $\begin{array}{llll}\text { Forestales, } & 9 & \text { (48), } & \text { 69-91. }\end{array}$ https://doi.org/10.29298/rmcf.v8i48.132

Golley, F. (1983). Decomposition. En F. Golley F. (Eds.), Tropical Rain Forest Ecosystems (pp. 157-166). Amsterdam, Netherland: Elsevier Scientific Publishing Company.

Goma-Tchimbakala, J. \& Bernhard-Reversat, F. (2006). Comparison of litter dynamics in three plantations of an indigenous timber-tree species (Terminalia superba) and a natural tropical forest in Mayombe, Congo. Forest Ecology and Management, 229(1-3), 304313.

Gómez, J. \& Sánchez, M. (1999). El proceso de descomposición de residuos vegetales. Universidad Nacional de Colombia, Palmira. Material para docencia, $12-14 \mathrm{pp}$.

González, G. \& Seastedt, T. R. (2001). Soil fauna and plant litter decomposition in tropical and subalpine forest. Ecology, 82(4), 955-964.

Hairiah, K., Sulistyani, H., Suprayogo, D., Purnomosidhi, P., Widodo, R. H., \& Van Noordwijk, M. (2006). Litter layer residence time in forest and coffee agroforestry systems in Sumberjaya, West Lampung. Forest ecology and management, 224(1-2), 45-57.

Instituto Nacional de Estadística y Geografía [Inegi]. (1986). Sintesis geográfica. Nomenclator y Anexo cartográfico del estado de Tabasco. 13 cartas temáticas (1:250 000). México, D. F.: Secretaría de Programación y Presupuesto.

Jeong, J., Kim, C., An, H. C., Cho, H. S. \& Choo, G. C. (2009). A comparison of litterfall dynamics in three coniferous plantations of identical age under similar site conditions. Journal of Ecology and Field Biology, 32(2), 97-102.
Lavelle, P., Blanchart, E., Martin, A., Martin, S., Spain, A., Toutain, F., Barois, I. \& Schaefer, R. (1993). A hierarchical model for decomposition in terrestrial ecosystems: application to soils of the humid tropics. Biotropica, 25(2), 130-150. doi: 10.2307/2389178

Martius, C., Höfer, H., Garcia, M. V., Römbke, J., \& Hanagarth, W. (2004). Litter fall, litter stocks and decomposition rates in rainforest and agroforestry sites in central Amazonia. Nutrient cycling in agroecosystems, 68(2), 137-154.

McDonald, M. A., \& Healey, J. R. (2000). Nutrient cycling in secondary forests in the Blue Mountains of Jamaica. Forest Ecology and Management, 139(1-3), 257-278. doi: 10.1016/S03781127(00)00442-4

Monedero, C., \& González, V. (1995). Producción de hojarasca y descomposición en una selva nublada del ramal interior de la cordillera de la costa, Venezuela. Ecotrópicos, 8(1-2), 1-14.

Neiff, J. J., \& de Neiff, A. P. (1990). Litterfall, leaf decomposition and litter colonization of Tessaria integrifolia (compositae) in the Paraná river floodplain. Hydrobiologia, 203(1-2), 45-52.

Olson, J. S. (1963). Energy storage and the balance of producers and decomposers in ecological systems. Ecology, 44(2), 322-331. https://doi.org/10.2307/1932179

Orihuela-Belmonte, D. E \& De Jong, B. H. J. (2012). Producción de hojarasca en la Reserva de la Biosfera Selva El Ocote, en el Estado de Chiapas. En F. Paz \& R. M. Cuevas (Eds.), Estado actual del conocimiento del ciclo del carbono y sus interacciones en México: Sintesis a 2011 (pp. 17-25). Texcoco, Estado de México, México: Universidad Autónoma del Estado de México-Programa Mexicano del Carbono-Instituto Nacional de Ecología.

Ortiz-Pérez, M. A, Siebe, C. \& Silke, S. (2005). Diferenciación ecogeográfica de Tabasco. En J. Bueno., F, Álvarez, \& S. Santiago (Eds.), Biodiversidad de estado de Tabasco (pp. 306-321). México: UNAM-Conabio.

Palacios-Bianchi, P. A. (2002). Producción y descomposición de hojarasca en un Bosque Maulino fragmentado. Seminario de título. Universidad de Chile. Santiago, Chile. Recuperado de http://www.mantruc.com/pilar/seminario-palaciosbianchi2002.pdf

Poggiani, F \& Schumacher, M. V. (2004). Nutrient cycling in native forests. En J. L. M. Goncalves \& V Benedetti (Eds.), Forest nutrition and fertilization (pp. 287-305). Piracicaba, Brasil: IPEF.

Polyakova, O. \& Billor, N. (2007). Impact of deciduous tree species on litterfall quality, decomposition rates and nutrient circulation in pine stands. Forest Ecology and Management, 253(1-3), 11-18. doi: 10.1016/j.foreco.2007.06.049 
Quinto, M. H., Ramos, P. Y. \& Abadía, B. D. (2007). Cuantificación de la caída de hojarasca como medida de la productividad primaria neta en un bosque pluvial tropical en Salero, Chocó, Colombia. Revista Institucional Universidad Tecnológica del Chocó D.L.C. 26, 28-41.

Ramírez, J. A., Zapata, C. M., León, J. D. \& González, M. I. (2007). Caída de hojarasca y retorno de nutrientes en bosques montanos andinos de piedras blancas, Antioquia, Colombia. Interciencia, 32(5), 303-311.

Ramírez, M. E. y Ataroff, M. (2001). Producción de hojarasca en una selva nublada andina: estacionalidad, descomposición y nitrógeno. Memorias del IV Simposio Internacional de Desarrollo Sustentable en Los Andes: la estrategia Andina para el siglo XXI. Recuperado de http://www.ciencias.ula.ve/icae/publicaciones/selvanublada/p df/ramirez2001a.pdf

Rave-Oviedo, S. Y., Montenegro-Ríos, M. \& Molina-Rico, L. J. (2013). Leaf fall and leaf litter decomposition of Juglans neotropica Diels (1906) (Juglandaceae) in an andean mountain forest, Pijao (Quindío), Colombia. Actualidades Biológicas, 35(98), 33-43.

Reyes-Carrera, S. A., Méndez-González, J., Nájera-Luna, J. A., \& Cerano-Paredes, J. (2013). Producción de hojarasca en un rodal de Pinus cembroides Zucc. en Arteaga, Coahuila, México y su relación con las variables climáticas. Revista Chapingo. Serie ciencias forestales y del ambiente, 19(1), 147-155.

Rivera-Vázquez, R., Soto-Pinto, L., Núñez-Colín, C. A., De Jung, B., Hernández-Rivera, M., \& Ordóñez-Díaz, J. A. (2013). Producción y tasa de descomposición de hojarasca en Acahuales de selva caducifolia en Chiapas. Revista mexicana de ciencias forestales, 4(20), 20-30.

Rocha-Loredo, A. G., \& Ramírez-Marcial, N. (2009). Producción y descomposición de hojarasca en diferentes condiciones sucesionales del bosque de pino-encino en Chiapas, México. Boletin de la Sociedad Botánica de México, 84, 1-12.

Rodríguez, E \& Rosas, M. (1993). Caída de hojarasca en el bosque alto andino de Carpanta. En G. Andrade (Eds.), "Carpanta" selva nublada y páramo: ecología y conservación de un ecosistema altoandino (1a ed.) (pp. 81-95). Fundación Natura, Bogotá, Colombia.

Rodríguez-Sandoval, J. J. (2012). Sucesión ecológica de un fragmento de selva mediana subperennifolia en la colonia La Cuchilla y la sabana de Quercus oleoides en el municipio de Balancán, Tabasco, México. Tesis de maestría. Universidad Juárez Autónoma de Tabasco. Villahermosa, Centro, Tabasco.

Sarmiento, G. (1984). Los ecosistemas y la ecósfera (1a ed.) (pp. 85-95). Barcelona, España: Editorial Blume.
Schessl, M., Da Silva, W. L., \& Gottsberger, G. (2008). Effects of fragmentation on forest structure and litter dynamics in Atlantic rainforest in Pernambuco, Brazil. Flora-Morphology, Distribution, Functional Ecology of Plants, 203(3), 215-228.

Singh, J. S., \& Gupta, S. R. (1977). Plant decomposition and soil respiration in terrestrial ecosystems. The botanical review, 43(4), 449528.

Swift, M. J. \& Anderson, J. M. (1989). Decomposition. En H. Lieth y M. J. A. Werger (Eds.), Tropical rain forest ecosystems. Biogeographical and Ecological Studies (pp. 547-569). Elsevier Science, Amsterdam.

Swift, M. J., Heal, O.W. \& Anderson, J. M. (1979). Decomposition in terrestrial ecosystems. Oxford: Blackwell, England.

Tudela, F. (Coord.) (1989). La modernización forzada del trópico: el caso de Tabasco. Proyecto Integrado del Golfo, México, D. F.: El Colegio de México, Cinvestav, IFIAS y UNRISD.

Veneklaas, E. J. (1991). Litterfall and nutrient fluxes in two montane tropical rain forests, Colombia. Journal of Tropical Ecology, 7(3), 319336. doi:10.1017/S0266467400005587

Villavicencio-Enríquez, L. (2012). Producción, pérdida de peso y tasas de descomposición de hojarasca en cafetales tradicional y rústico, y selva mediana, en Veracruz, México. Revista Chapingo. Serie ciencias forestales y del ambiente, 18(2), 159-173. Recuperado de https:/ / www.redalyc.org/articulo.oa?id=629/62924540002

Vitousek, P. M., \& Sanford Jr, R. L. (1986). Nutrient cycling in moist tropical forest. Annual review of Ecology and Systematics, 17(1), 137-167.

Wang, Q., Wang, S., Fan, B., \& Yu, X. (2007). Litter production, leaf litter decomposition and nutrient return in Cunninghamia lanceolata plantations in south China: effect of planting conifers with broadleaved species. Plant and Soil, 297(1-2), 201-211. doi: 10.1007/s11104-007-9333-2

Xuluc-Tolosa, F. J., Vester, H. F. M., Ramırez-Marcial, N., CastellanosAlbores, J., \& Lawrence, D. (2003). Leaf litter decomposition of tree species in three successional phases of tropical dry secondary forest in Campeche, Mexico. Forest Ecology and Management, 174(13), 401-412. doi: 10.1016/S0378-1127(02)00059-2.

Manuscrito recibido el 15 de diciembre de 2017

Aceptado el 7 de julio de 2019

Publicado el 17 de marzo de 2020 
Este documento se debe citar como:

García-Domínguez, A., Cámara C., L. C., van der Wal, J. C., \& Martínez S., J. L. (2020). Producción y tasa de descomposición de hojarasca en acahuales de Tabasco, México. Madera y Bosques, 26(1), e2611694. doi: 10.21829/myb.2020.2611694
Madera y Bosques por Instituto de Ecología, A.C. se distribuye bajo una Licencia Creative Commons Atribución-NoComercialCompartirlgual 4.0 Internacional. 